Génét. Sél. Evol., 1988, 20 (3), 387-396

\title{
Note
}

\section{Lignées divergentes pour la consommation alimentaire "résiduelle " des pondeuses : réponse des poussins à une inoculation par Eimeria acervulina et comparaison de paramètres biologiques}

\author{
Jessica KATLE *, Nicole HAMET **, L. DURAND ***, \\ P. ROMBAUTS ${ }^{* * * *}$ et P. MÉRAT $* * *$ \\ * Norges Landbrukshøgskole, Institut for fjørfe og pelsdyr \\ Boks 17, 1432 AS-NLH, Norvège \\ ** Ministère de l'Agriculture, Direction de la Qualité, Institut d'Elevage, \\ de Pathologie et d'Hygiène alimentaire, Laboratoire de Parasitologie, 22440 Ploufragan, France \\ *** Institut National de la Recherche Agronomique, Centre de Recherches de Jouy-en-Josas, \\ Laboratoire de Génétique factorielle, 78350 Jouy-en-Josas, France \\ **** Institut National de la Recherche Agronomique, Centre de Recherches de Jouy-en-Josas, \\ Laboratoire de Physiologie Animale, 78350 Jouy-en-Josas, France
}

\section{Résumé}

Un total de 343 poussins appartenant à deux lignées, $R^{+}$et $R^{-}$, respectivement sélectionnées pour une forte ou faible composante "résiduelle" de la consommation alimentaire au stade adulte, a été réparti dans un lot témoin et un lot inoculé à l'âge de 25 jours par $10^{6}$ ookystes d'Eimeria acervulina (agent de la coccidiose duodénale). L'abattage avait lieu sept jours après l'inoculation. La variation de poids, la consommation alimentaire pendant la période expérimentale, l'excrétion des ookystes, l'intensité des lésions et d'autres signes pathologiques, ainsi que divers paramètres sériques (glucose, protéines, enzymes, corticostérone) étaient mesurés. Outre la diminution de croissance, de consommation et d'efficacité alimentaire et la production de lésions caractéristiques, les effets de l'inoculation par $E$. acervulina sur les paramètres sanguins enregistrés sont brièvement discutés. On note une interaction complexe entre sexe et traitement pour le glucose et la phosphatase alcaline, ce qui n'est pas le cas pour le gain de poids et l'intensité des lésions. En réponse au traitement, les deux lignées ne diffèrent ni pour la variation de poids, ni pour l'ingestion alimentaire; la distribution de la note de lésions suggère une valeur moyenne un peu plus grande dans la lignée $R^{-}$pour laquelle, d'autre part, les globulines $\alpha_{2}$ augmentent davantage sous l'effet du traitement. Indépendamment de la réponse à l'agent pathogène, les deux lignées diffèrent par le taux de protéines totales du sérum (d'environ $10 \%$ plus élevé pour $R^{+}$ dans chaque traitement), pour le taux de corticostérone (plus élevé dans $R^{+}$, surtout en lot témoin). Le glucose a une valeur globalement plus élevée et le taux d'estérase est notablement plus faible dans la lignée $R^{-}$, mais en interaction avec les facteurs sexe et traitement. Le taux de caeruloplasmine montre une interaction de la lignée avec le traitement, et la phosphatase alcaline une interaction lignée $\times$ sexe.

Mots clés: sélection, efficacité alimentaire, différences entre lignées, coccidiose, paramètres biochimiques. 


\section{Summary \\ Divergent lines for " residual " feed intake of layers : response of chicks to inoculation by Eimeria acervulina and comparison of biological parameters}

Three hundred and forty three chicks in total, belonging to two lines, $R^{+}$and $R^{-}$, respectively bred for a high and a low "residual » component of feed intakc at the adult stage, were distributed in a control group and a group inoculated at the age 25 days with $10^{6}$ oocysts of Eimeria acervulina (duodenal coccidiosis). The birds were slaughtered seven days after the inoculation. The body weight variation, the feed intake during the experimental period, oocyst excretion, lesion score and other clinical signs, together with several blood parameters (glucose, proteins, enzymes, corticosterone) were measured. In addition to the depression of growth rate, feed intake and feed efficiency and the appearance of characteristic lesions, the effect of the inoculation by $E$. acervulina on the recorded blood parameters are briefly discussed. A complex interaction between sex and treatment is noted for glucose and alkaline phosphatase. On the contrary, for body weight gain and lesions, no such interaction is observed. For their response to treatment, the two lines differ neither for body weight variation, nor for feed consumption; the distribution of the lesion score suggests a slightly higher mean value in the $R^{-}$line. In this line, on the other hand, the $\alpha_{2}$-globulins increase more in response to the treatment than in the $R^{+}$line. Independently of the response to the pathogen, the lines differ in the level of total proteins in the serum (higher in $R^{+}$by about $10 \%$ in both treatments), in the level of corticosterone (higher in $R^{+}$, mainly in the control). The glucose on the whole has a higher value and the esterase has a noticeably lower value in the $R^{-}$line, but with interactions with sex and treatment. The caeruloplasmin level shows a line $\times$ treatment interaction and alkaline phosphatase shows a line $\times$ sex interaction. meters.

Key words : selection, feed efficiency, differences between lines, coccidiosis, biochemical para-

\section{Introduction}

Deux lignées sélectionnées en sens contraire sur la composante « résiduelle » de la consommation alimentaire des coqs et poules adultes (écart de la consommation observée à celle prédite à partir du poids corporel et de la production d'œufs : cf. Bordas \& MÉrat, 1984a) ont présenté une différence significative pour la mortalité des poulettes après l'âge de 18 semaines, avec un taux de survie de $3 \%$ supérieur en moyenne dans la lignée à consommation plus élevée.

Nous avons voulu comparer la réponse de ces lignées à un parasite entraînant une morbidité sans mortalité. Nous avons choisi d'inoculer des poussins des deux lignées par Eimeria acervulina, agent responsable de la coccidiose duodénale. Plusieurs paramètres physiologiques et biochimiques ont été mesurés, en même temps que les critères zootechniques et pathologiques.

\section{Matériel et méthodes}

\section{A. Matériel animal}

Les lignées $R^{+}$et $\mathrm{R}^{-}$, issues d'une population de base Rhode-Island et sélectionnées comme indiqué ci-dessus, ont été décrites par Bordas \& MÉrat (1984a, et 1984b). 
Dans une éclosion pedigree d'œufs collectés durant trois semaines, en 1986, à partir de 9 pères par lignée et 5 mères par père, 343 poussins ont été obtenus, déduction faite d'une faible mortalité non spécifique survenue entre l'éclosion et la fin de l'expérience (2 morts).

\section{B. Lots, conditions expérimentales}

Les poussins ont été envoyés à l'Insitut d'Elevage, de Pathologie et d'Hygiène alimentaire à Ploufragan. A l'intérieur de chaque lignée, sexe et famille de père, ils ont été répartis en deux lots, témoin $(\mathrm{T})$ et contaminé $(\mathrm{C})$, en égalisant autant que possible le poids moyen dans chaque lot. Les poussins étaient placés dans des cages, dont chacune contenait de 2 à 4 animaux du même sexe et de la descendance du même père. L'aliment contenait approximativement $18 \%$ de protéines totales et $2850 \mathrm{kcal} / \mathrm{kg}$ d'énergie métabolisable. L'inoculation de l'agent infectieux était faite à 25 jours d'âge. La dose inoculée d'Eimeria acervulina était $10^{6}$ ookystes par animal. Les animaux étaient abattus 7 jours après l'inoculation.

\section{Observations et mesures}

\section{Croissance pondérale et consommation d'aliment}

Les oiseaux étaient pesés individuellement la veille de l'inoculation, puis 7 jours après l'inoculation, avant l'abattage. Leur variation de poids durant la période de 8 jours correspondante en était déduite. La consommation d'aliment pendant la même période était enregistrée par cage. On en déduisait la consommation moyenne et l'indice de consommation moyen (consommation/gain de poids) par individu pour chaque cage.

\section{Variables caractérisant l'état pathologique}

Aucune mortalité n'était attribuable à l'inoculation. La gravité des lésions intestinales était notée à l'abattage selon l'échelle de Long \& ReID (1982).

Les mesures ci-après étaient faites par groupes de 4 poussins élevés dans la même cage, sur un échantillon de 9 cages par lignée :

— note caractérisant l'aspect des matières fécales $(0$ : normales $; 1:$ molles : 2 : très molles ; 3 : liquides non glaireuses ; 4 : très liquides avec mucosités) ; trois observations étaient faites, respectivement 4,5 et 6 jours après inoculation ;

- note de morbidité $(0$ : attitude normale ; 1 : plumes ébouriffées, surtout du cou ; 2: début de la frilosité et de la prostation; 3 : frilosité et prostation plus marquées ; 4 : plumes toutes ébouriffées, position "en boule ", ailes tombantes, yeux fermés, station debout pénible) ; observation répétée également à 4,5 et 6 jours après l'inoculation ; 
- évaluation de l'excrétion ookystale moyenne par animal, du $4^{\mathrm{e}}$ au $6^{\mathrm{e}}$ jour après l'inoculation, selon la technique décrite par HAMET (1983).

\section{Paramètres biochimiques}

A partir d'une prise de sang intra-cardiaque, faite avant l'abattage sur tous les animaux, les paramètres biochimiques suivants étaient mesurés dans le plasma sanguin :

- glucose ;

- carboxyl estérase (méthode décrite par GaSPARSKA \& JASTRzebSKI, 1984) ;

- phosphatase alcaline (méthode décrite par BESSEY et al., 1946) ;

- caeruloplasmine (méthode de Houchin, 1958, modifiée par RICE, 1960) ;

- corticostérone (sur un échantillon de 30 poussins femelles pris au hasard dans chaque lignée et traitement) par dosage radioimmunologique à l'aide d'un anti-sérum anticorticostérone-21-thyréoglobuline (ETCHEs, 1976 ; RombaUTs et al., 1983) ;

- protéines du sérum (sur un échantillon de 117 mâles pris au hasard, 27 à 30 par lignée et traitement) ; après séparation par électrophorèse sur gel de polyacrylamide, une lecture de densité optique (densitomètre Vernon) a été faite pour estimer le pourcentage des classes suivantes (BUTLER, 1971) : sérumalbumine, globulines $\alpha_{1}$ et $\alpha_{2}$, transferrines; le reste des protéines migrant après les transferrines était évalué par différence. Les protéines totales étaient estimées d'autre part par la réaction du biuret.

\section{Analyses statistiques}

Les variables mesurées individuellement dans les deux groupes expérimentaux (poids et sa variation, glucose, estérase, phosphatase alcaline, caeruloplasmine) ont fait l'objet d'une analyse de variance à trois facteurs (traitement, sexe, lignée) tenant compte des effectifs inégaux dans les sous-classes (SNEdecor \& Cochran, 1969).

La consommation alimentaire, relevée par cage, a été analysée selon les facteurs traitement, sexe et lignée, de même que l'indice de consommation.

Le taux de corticostérone plasmatique, mesuré seulement sur un échantillon de 120 femelles, a fait l'objet d'une analyse séparée (facteurs traitement et lignée). Il en est de même des composantes des protéines du sérum, mesurées seulement sur 117 mâles.

La note de lésions a été soumise à une analyse de variance à deux facteurs (lignée, sexe) dans le lot contaminé ; nous avons d'autre part fait une comparaison, par test de $\chi^{2}$, de la fréquence dans les lignées $R^{+}$et $R^{-}$des diverses notes (1 et 2 étant regroupées).

Les notes caractérisant les matières fécales et la morbidité et le comptage des ookystes, établis sur 9 cages par lignée, ont été comparées pour les deux lignées par un test $t$.

\section{Résultats et discussion}

Le tableau 1 donne les valeurs moyennes, par traitement, sexe et lignée, de toutes les variables, à l'exception de celles mesurées seulement sur 9 cages par lignée. Pour 


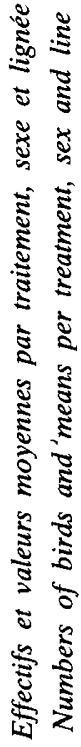

\begin{tabular}{|c|c|c|c|c|c|c|c|c|}
\hline \multirow{8}{*}{ 套 } & \multirow{2}{*}{ 粟 } & $\approx$ & ్ి & $\stackrel{m}{\vec{\nu}} \stackrel{m}{2}$ & $\ddot{0}$ & 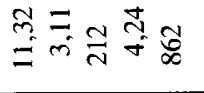 & $\stackrel{\Delta}{\Xi}$ & \\
\hline & & $\stackrel{+}{ \pm}$ & ஓ & $\stackrel{2}{\circ} \tilde{n}^{n}$ & to & 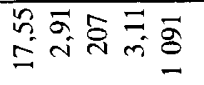 & $\hat{m}$ & \\
\hline & \multirow{2}{*}{ 퐁 } & ot & $\stackrel{\infty}{=}$ & $\bar{\Xi} \stackrel{0}{0}$ & $\begin{array}{l}0 \\
\stackrel{\infty}{ \pm} \\
\dot{J}\end{array}$ & 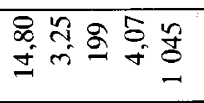 & ?n & \\
\hline & & 8 & $\overline{\vec{\sim}}$ & ల్ $\stackrel{\infty}{=}$ & $\stackrel{0}{\underline{N}}$ & 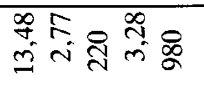 & $\ddot{n}$ & \\
\hline & \multicolumn{2}{|c|}{$\begin{array}{l}1 \\
\frac{1}{2} \\
\alpha+z \\
z\end{array}$} & $\underset{\infty}{=}$ & $\stackrel{\sigma}{\sigma}$ & $\stackrel{0}{\stackrel{2}{2}}$ & 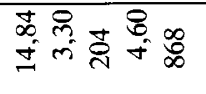 & $\begin{array}{ll}a & 2 \\
n & m \\
n & m\end{array}$ & \\
\hline & \multicolumn{2}{|c|}{$\frac{+F}{a+z}$} & 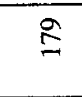 & $\stackrel{2}{=}$ & 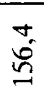 & 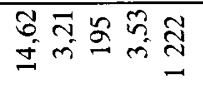 & $\begin{array}{l}2 \\
\pm \\
\pm\end{array}$ & \\
\hline & \multicolumn{2}{|c|}{ 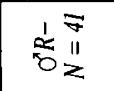 } & $\stackrel{\circ}{\sim}$ & $\ddot{\sim} \cong$ & $\stackrel{\infty}{\infty}$ & 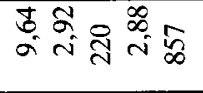 & $\stackrel{0}{2} 1$ & 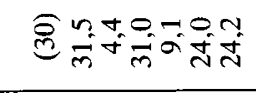 \\
\hline & \multicolumn{2}{|c|}{ 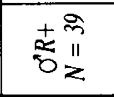 } & $\stackrel{\infty}{\vec{N}}$ & త్రి & $\stackrel{\substack{n \\
\infty}}{\infty}$ & 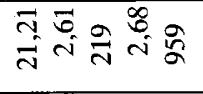 & 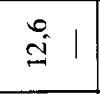 & 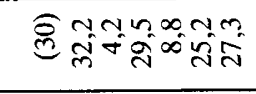 \\
\hline \multirow{8}{*}{ 梽 } & \multirow{2}{*}{ 㶾 } & $\propto$ & $\lesssim$ & ळે ڤે & जे & $\stackrel{\infty}{m} \mid \stackrel{m}{\sim} \stackrel{d}{\dot{n}}$ & $\begin{array}{l}0 \\
\dot{y}\end{array}$ & \\
\hline & & $\stackrel{+}{a}$ & $\stackrel{\infty}{\varrho}$ & $\ddot{d} \underset{\infty}{\infty}$ & ڤֶ & $\bar{m} \mid \hat{a} \hat{n} \infty$ & क & \\
\hline & \multirow{2}{*}{ 粟 } & O+ & & 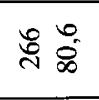 & $\hat{n}$ & 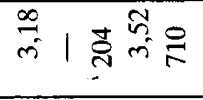 & సi & \\
\hline & & 8 & $\stackrel{\ominus}{\sim}$ & \&్లి & 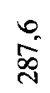 & 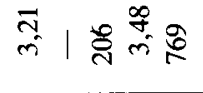 & $\ddot{8}$ & \\
\hline & \multicolumn{2}{|c|}{$\begin{array}{l}1 \approx \pi \\
\approx+11 \\
\alpha+z\end{array}$} & $\bar{\infty}$ & హి & 牯 & 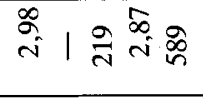 & त̂ & \\
\hline & \multicolumn{2}{|c|}{ 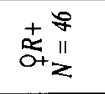 } & $\begin{array}{l}\infty \\
\infty\end{array}$ & స్ & 吕 & $\stackrel{n}{m} \mid \stackrel{\infty}{*} \bar{\sim}$ & $\begin{array}{l}\infty \\
m \\
m\end{array}$ & \\
\hline & \multicolumn{2}{|c|}{$\begin{array}{ll}\frac{1}{12} \\
\frac{2}{0} \\
0 \\
z\end{array}$} & $\stackrel{m}{\sim}$ & ઠิ & ले & $\stackrel{\infty}{m} \mid \stackrel{\vec{n}}{\text { m̊g }}$ & $\therefore 1$ & 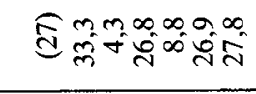 \\
\hline & \multicolumn{2}{|c|}{ 等 } & 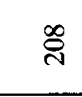 & $\stackrel{\bar{े}}{\hat{\infty}}$ & ڤి & సু। & $\stackrel{\infty}{\forall} \mid$ & 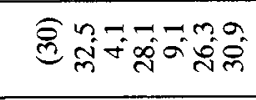 \\
\hline \multicolumn{3}{|c|}{. } & 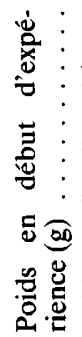 & 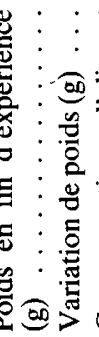 & & 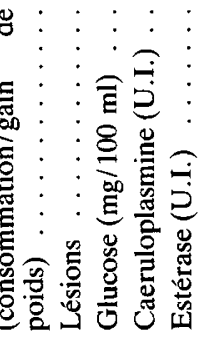 & 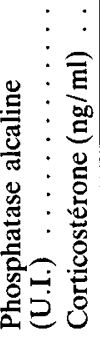 & 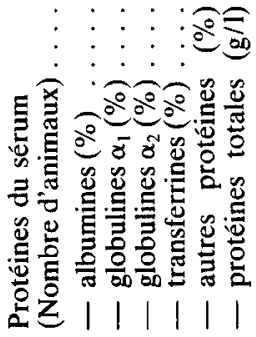 \\
\hline
\end{tabular}




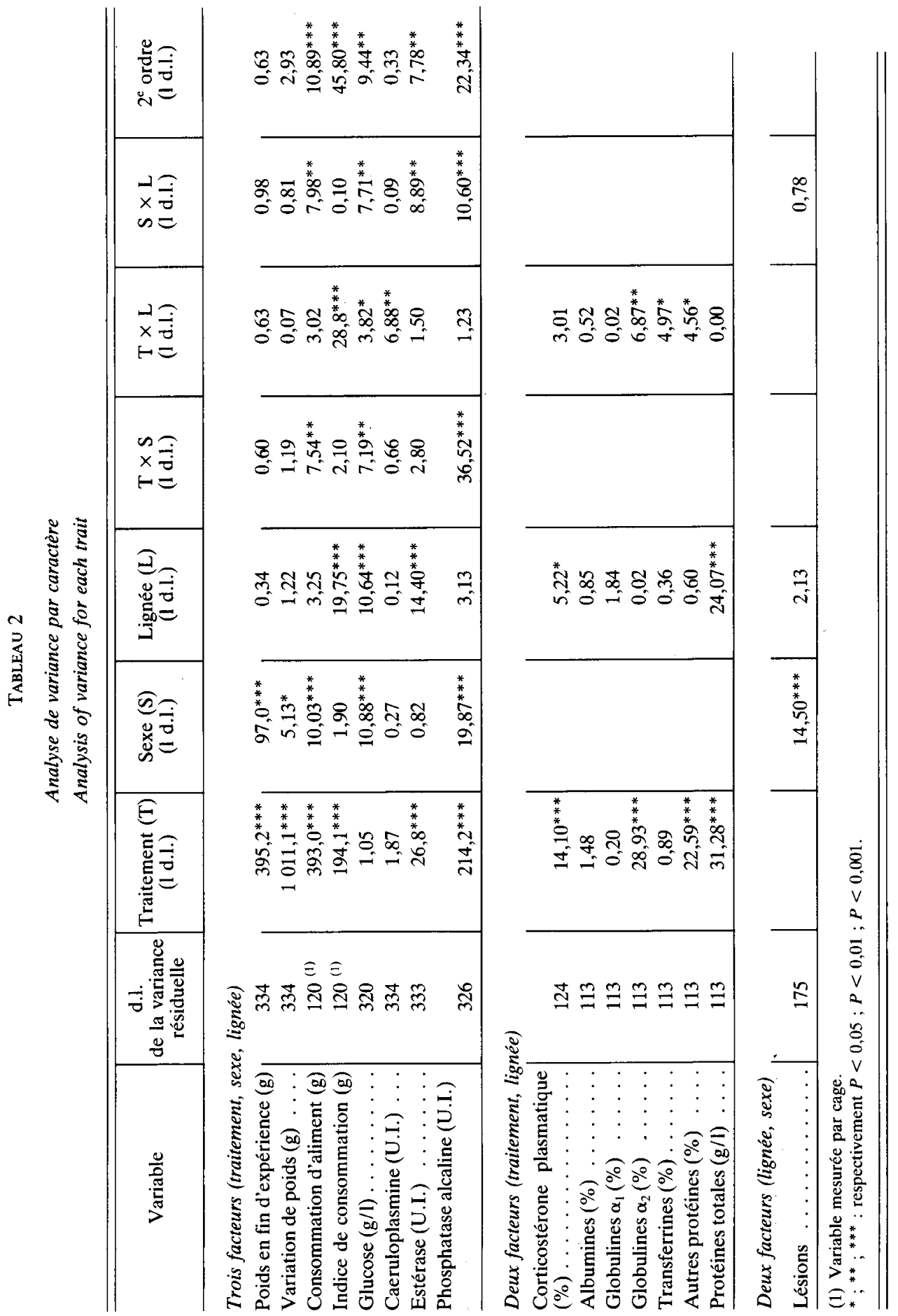


ces dernières, les valeurs moyennes respectives de $R^{+}$et $R^{-}$sont : 3,56 et 3,78 puis 0,78 et 0,78 pour les matières fécales respectivement à $\mathrm{J}+4$ et $\mathrm{J}+5 ; 3,44$ et 3,89 pour la morbidité $(\mathrm{J}+4) ; 1,10$ et 1,22 (morbidité $: \mathrm{J}+5) ; 18,4$ et $11,9 \times 10^{6}$ pour le nombre d'ookystes excrétés de $\mathrm{J}+4$ à $\mathrm{J}+6$. Aucune différence significative entre lignées n'apparaît.

Pour les variables du tableau 1, le tableau 2 présente l'analyse de variance selon les trois facteurs «traitement", "sexe » et "lignée » ou selon les deux facteurs « traitement » et « lignée » ou "sexe » et « lignée » suivant le cas.

Au tableau 3 figure le détail de la répartition des notes caractérisant les lésions dans les lignées $\mathbf{R}^{+}$et $\mathbf{R}^{-}$.

\section{TABleau 3}

Répartition des notes caractérisant les lésions par lignée (sexes regroupés) Distribution of the lesion scores according to the line (sexes pooled)

\begin{tabular}{c|c|c|c|c|c|c}
\hline \multirow{2}{*}{ Note } & \multicolumn{5}{|c}{ Nombre d'individus par lignée et pourcentage du total } \\
\cline { 2 - 7 } & $\begin{array}{c}R^{-} \\
\text {Nombre }\end{array}$ & $\%$ & $\begin{array}{c}R^{+} \\
\text {Nombre }\end{array}$ & $\%$ & \multicolumn{2}{|c}{ Total } \\
\cline { 5 - 7 } & 30 & 32,3 & 30 & 35,7 & 60 & 33,9 \\
\hline ou 2 & 19 & 20,4 & 28 & 33,3 & 47 & 26,6 \\
3 & 44 & 47,3 & 26 & 31,0 & 70 & 39,5 \\
\hline 4 & 93 & & 84 & & 177 & \\
\hline Total & & & & & & \\
\hline
\end{tabular}

$\chi^{2} 2 \mathrm{ddl}=5,89(\mathrm{P}<0.05)$

\section{A. Effet global du traitement}

Comme on pouvait s'y attendre, l'inoculation par Eimeria acervulina a occasionné des lésions caractéristiques et une réduction hautement significative de la croissance pondérale et de la consommation alimentaire. Quant aux paramètres biochimiques, le glucose n'est pas significativement modifié, mais la phosphatase alcaline est diminuée de façon hautement significative. Ces résultats sont conformes à la description par Yvoré et al. (1972) des effets de la maladie. Par comparaison avec l'effet d'autres types de coccidiose (WITLOCK et al., 1981 ; WITLOCK, 1984), on n'observe pas ici de diminution des réserves glucidiques. L'estérase plasmatique est très significativement augmentée chez les animaux inoculés par rapport aux témoins. Ce résultat ne semble pas avoir été signalé et son interprétation nécessiterait d'autres éléments sur le rôle physiologique de l'enzyme concernée. Enfin, la contamination fait baisser dans les deux lignées le taux de corticostérone plasmatique : l'effet de l'infection par $E$. acervulina n'est pas assimilable à cet égard à un " stress ». Ceci diffère des observations de CHALley (1966) dans une infection par Eimeria tenella. Peut-être en relation avec l'absence d'augmentation de la corticostérone, la caeruloplasmine n'est pas augmentée par le traitement, alors qu'un accroissement important de cette enzyme a été signalé sous l'effet de ACTH qui stimule les surrénales. 
Les protéines totales et les albumines du sérum sont très significativemeṇt abaissées à la suite de l'inoculation, de même que dans les observations de Yvorḱ et al. (1972), RufF \& Augustine (1982) sur poulets et de Augustine (1985) sur dindons. Ceci peut concorder avec la baisse de consommation alimentaire et la mauvaise utilisation des protéines observée par LARBIER et al. (1974). En pourcentage, la seule classe qui soit irès significativement augmentée par le traitement est celle des $\alpha_{2}$-globulines (tableau 1), dont la nature ne paraît pas précisée (BuTler, 1971). Une augmentation semblable a été signalée chez le dindon après une infection par Eimeria (Augustine, 1985) alors que sur les poulets infectés par E. acervulina, RufF \& Augustine (1982) ne trouvent pas d'accroissement net de cette fraction. Corrélativement dans nos données, les globulines faisant suite aux transferrines diminuent en proportion. Le délai après l'inoculation apparaît court pour que des immunoglobulines interviennent de façon appréciable dans l'une de ces fractions.

\section{B. Effet du sexe}

Outre le poids corporel et la consommation alimentaire, il existe un effet du sexe sur le glucose et la phosphatase alcaline, mais en interaction complexe avec le traitement ; les différences entre sexes sont plus nettes en lot contaminé pour le glucose (moyenne plus élevée des mâles) et en lot témoin pour la phosphatase alcaline.

La réponse des deux sexes à la contamination n'apparaît pas sensiblement différente ; l'interaction sexe $\times$ traitement pour la consommation alimentaire ou pour le glucose ne correspond pas à des écarts importants dans les valeurs moyennes ; il n'y a que la phosphatase alcaline pour laquelle une différence marquée entre sexes en lot témoin est supprimée en lot contaminé.

\section{Effet de la lignée et interactions}

Pour leur réponse au traitement, les lignées, dans l'ensemble, ne diffèrent de façon nette ni pour la variation de poids, ni pour l'ingestion alimentaire, ni pour la note de lésions. Pour cette dernière, cependant, le $\chi^{2}$ tiré de la distribution des valeurs par lignée suggère une intensité un peu plus grande des lésions dans la lignée $R^{-}$, mais cet effet isolé demande à être confirmé. Quant à l'indice de consommation, il est difficilement interprétable chez les animaux contaminés, leurs gains de poids étant très faibles ou nuls.

Indépendamment de la réponse à l'agent pathogène étudié, la différence entre les lignées $R^{+}$et $R^{-}$pour le taux de corticostérone plasmatique suggère que la lignée $R^{+}$, consommatrice d'aliment moins efficace à l'âge adulte, est plus sensible au léger stress causé par une manipulation des animaux. Le taux de glucose globalement plus élevé et le taux d'estérase notablement plus faible dans la lignée $R^{-}$restent à analyser quant à leur signification et quant à leur interprétation éventuelle comme réponse liée à la sélection exercée dans ces lignées. Pour ces deux dernières variables, le tableau est d'ailleurs compliqué par une interaction de la lignée avec le sexe, et avec le traitement pour le glucose, et, dans les deux cas, une interaction du deuxième ordre. Cependant, 
le niveau de l'estérase est partout supérieur pour la lignée $R^{+}$, sauf chez les mâles en lot témoin. D'autres variables, sans différer significativement dans l'ensemble selon la lignée, montrent une interaction entre lignée et traitement (caeruloplasmine), entre lignée et sexe (consommation alimentaire, phosphatase alcaline); il n'est pas possible pour l'instant de les interpréter et les différences correspondantes sont relativement limitées.

Sur la quantité totale des protéines du sérum, il y a un effet hautement significatif de la lignée : quel que soit le traitement, la lignée $R^{+}$en contient environ $10 \%$ de plus que $R^{-}$. Par contre, il n'y a pas d'effet de la lignée sur la proportion des diverses protéines, mais une interaction hautement significative apparaît entre lignée et traitement pour les globulines $\alpha_{2}$. Celles-ci augmentent plus, sous l'effet du traitement, pour la lignée $R^{-}$que pour la lignée $R^{+}$, proportionnellement et en valeur absolue. Une comparaison de leur pourcentage entre lots témoin et inoculé dans la seule lignée $R^{-}$, par un test $t$, montre d'ailleurs une différence hautement significative $(t=6,64$; $P<0,001)$ alors que la différence analogue dans la lignée $R^{+}$n'atteint pas le seuil $5 \%$ de signification, et qu'en lot témoin seul les deux lignées ne diffèrent pas significativement l'une de l'autre. Il est prématuré d'interpréter cette différence de réaction des deux lignées vis-à-vis d'Eimeria acervulina, un rapprochement avec les autres différences de réponses notées (lésions, glucose, caeruloplasmine) n'étant pas évident.

\section{Conclusion}

Les lignées comparées ne diffèrent donc pas notablement pour leur sensibilité à Eimeria acervulina. Deux indices, cependant, d'une légère différence dans leur réponse sont l'intensité des lésions, un peu plus grande dans $R^{-}$d'après le test $\chi^{2}$, et la présence d'une interaction traitement $\times$ lignée hautement significative pour les globulines $\alpha_{2}$ dont la proportion dans le sérum augmente davantage dans la lignée $R^{-}$avec la contamination. Concernant certains autres paramètres biochimiques, l'interaction observée ne correspond qu'à une faible différence dans la variation des moyennes selon le traitement.

Reçu le 4 mai 1987. Accepté le 8 décembre 1987.

\section{Références bibliographiques}

Augustine P.C., 1985. Electrophoretic separation of serum proteins and lipoproteins of young turkeys infected with Eimeria meleagrimitis or Eimeria adenoeides. Poult. Sci., 64, 1644-1648.

BeSSEY O.A., Lowry O.H., Brock M.J., 1946. A method for the rapid determination of alkaline phosphatase with five cubic millimeters of serum. J. Biol. Chem., 164, 321-329.

Bordas A., Mérat P., 1984a. Réponses liées dans une expérience de sélection sur la consommation alimentaire "résiduelle " de coqs et poules Rhode-Island. In : $17^{\mathfrak{T}}$ Congrès mondial d'aviculture, Helsinki, 8-12 août 1984, 106-108. 
Bordas A., Mérat P., 1984b. Correlated responses in a selection experiment on residual feed intake of adult Rhode Island red cocks and hens. Ann. Agric. Fenn., 23, 233-237.

Butler E.J., 1971. Plasma proteins. In : Bell D.J., Freeman B.M. (ed.), Physiology and biochemistry of the domestic fowl, vol. 2, 933-962, Academic Press, London.

Challey J.R., 1966. Changes in adrenal constituents and their relationship to corticosterone secretion in chickens selected for genetic resistance and susceptibility to cecal coccidiosis. $J$. Parasitol., 52, 967-974.

Etches R.J., 1976. A radio immuno assay for corticosterone and its application to the measurement of stress in poultry. Steroids, 28, 763-773.

Gasparka J., JastrzebskI Z., 1984. Blood plasma carboxylesterase activity in chicken. Pr. Mater. Zootech., 33, 7-15.

Hamet N., 1983. Techniques de contrôle de la présence d'ookystes de coccidies dans les matières fécales de volailles. In : 3rd International symposium for veterinary laboratory diagnosticians. Ames, lowa, June 13-15, 1985, 6 p., World Association of veterinary laboratory diagnosticians.

HouchiN O.B., 1958. A rapid colorimetric method for the quantitative determination of copper oxidase activity (caeruloplasmin). Clin. Chem., 4, 519-523.

Larbier M., Yvoré P., Guillaume J., 1974. Influence de la coccidiose duodénale sur l'utilisation de l'énergie et des protéines alimentaires chez le poulet. Ann. Rech. Vét., 5, 179-188.

LoNG P.L., REID M.W., 1982. A guide for the diagnosis of coccidiosis in chickens. Research Report 404, The University of Georgia college of Agriculture Experiment Station.

RicE E.W., 1960. Correlation between serum copper, caeruloplasmin activity and c-reactive protein. Clin. Chem. Acta, 5, 632-636.

Rombauts P., Bordas A., Banerjee A.K., Mérat P., 1983. Taux de corticostérone plasmatique, variation de poids et de température corporelle de poules Leghorn blanches naines $(d w)$ et normales $\left(\mathrm{Dw}^{+}\right)$en réponse à une privation d'aliment. Génét. Sél. Evol., 15, 251-256.

Ruff M.D., Augustine P.C., 1982. Effects of coccidiosis on the electrophoretic pattern of serum proteins in chickens. J. Parasitol., 68, 107-111.

Snedecor G.W., Cochran W.G., 1969. Statistical methods. 6th ed., 607 p., Iowa State University Press, Ames.

WITLOck D.R., 1984. Changes in moribund chickens as a result of Eimeria necatrix infection. J. Parasitol., 70, 433-435.

Witlock D.R., Ruff M.D., Chute M.B., 1981. Physiological basis of Eimeria tenella induced mortality in individual chicken. J. Parasitol., 67, 65-69.

Yvoré P., Dubois M., Sauveur B., Aycardi J., 1972, Pathogénie de la coccidiose duodénale à Eimeria acervulina. Ann. Rech. Vét., 3, 61-82. 